PROCEEDINGS OF THE

AMERICAN MATHEMATICAL SOCIETY

Volume 136, Number 10, October 2008, Pages 3375-3382

S 0002-9939(08)09547-6

Article electronically published on May 28, 2008

\title{
DOWN-UP ALGEBRAS AT ROOTS OF UNITY
}

\author{
RAJESH S. KULKARNI
}

(Communicated by Martin Lorenz)

\begin{abstract}
In this note, we show that down-up algebras at roots of unity are maximal orders over their centers.
\end{abstract}

\section{INTRODUCTION}

Down-up algebras were defined by Benkart and Roby ([2]) for combinatorial reasons. They are associative algebras $A=A(\alpha, \beta, \gamma)$ generated by $u, d$ over $\mathbb{C}$ and satisfy the relations

$$
\begin{aligned}
& d^{2} u=\alpha d u d+\beta u d^{2}+\gamma d, \\
& d u^{2}=\alpha u d u+\beta u^{2} d+\gamma u,
\end{aligned}
$$

where $\alpha, \beta, \gamma \in \mathbb{C}$. These have been studied by various authors, for example, in [2, 3, 5, 6, 7. We consider the case when $\beta \neq 0$, so that the algebra $A$ is Noetherian (6]). These algebras have a rich representation theory; see [2, 7]. We recall some basic structural properties.

Let $\xi=u d, \eta=d u$. Then $\xi \eta=\eta \xi$. Consider the automorphism $\theta$ of $\mathbb{C}[\xi, \eta]$ given by

$$
\theta(\xi)=\frac{\eta-\alpha \xi-\gamma}{\beta}, \quad \theta(\eta)=\xi
$$

Then $u r=\theta(r) u, d r=\theta^{-1}(r) d$ for any $r \in \mathbb{C}[\xi, \eta]$. This observation makes $A$ into a hyperbolic ring $([7])$ or a generalized Weyl algebra ([6]). Also, it is easy to see that $\mathbb{C}[\xi, \eta]$ embeds into $A$. We recall two other basic facts about this family of algebras: $A$ has a PBW-basis $\left\{u^{i}(d u)^{j} d^{k}\right\}([2,7])$, and $A$ is a domain if and only if $\beta \neq 0$. (6, 7]). The first property has been exploited in studying representations when the parameters are generic. We will use both of these properties in later sections.

In [7, we also studied the case when the automorphism $\theta$ has finite order. We call these algebras down-up algebras at roots of unity; see Section 2 below for details. They have been of recent interest; see, for example, [3, 5]. In [7], we showed that certain localizations of the algebra $A$ are crossed product algebras. We also showed that away from a subvariety of the variety defined by the center, the algebra is Azumaya. This together with other results from [7] raises a natural question: is the algebra $A$ at roots of unity a maximal order over its center? In this article, we prove that the answer is affirmative. In fact, first we prove that the down-up algebra

Received by the editors July 5, 2006.

2000 Mathematics Subject Classification. Primary 16H05; Secondary 17B37.

The author was partially supported by NSF grants DMS-0311850 and DMS-0603684.

(C)2008 American Mathematical Society 3375

Reverts to public domain 28 years from publication 
$A$ is a reflexive order in the central simple algebra $A \otimes K$ where $K$ is the fraction field of the center of $A$ (which is a domain). This result is then used in proving the main result. The technique for this main result should be useful in other situations as well. We use some old theorems of M. Harada and S. Williamson (4, 10]) which give a criterion for a crossed product algebra over a discrete valuation ring to be a maximal order. These theorems together with characterization of maximal orders due to Auslander and Goldman are the main tool used below.

Now we sketch a different approach for the main question of this article. It uses a result of J. T. Stafford (Theorem 2.10, 9]). In our context, this result says that a Noetherian, Auslander-regular and Cohen-Macaulay $k$-algebra over a field $k$ which is stably free is a domain and a maximal order in its quotient division ring. For the notion of a maximal order in this theorem, refer to 5.1.1, 8]. Now by Lemma 4.2, [6], the down-up algebra $A$ (when $\beta \neq 0$ ) is Auslander-regular and Cohen-Macaulay. Since a graded ring is stably free, the theorem cited earlier shows that the algebra $A=A(\alpha, \beta, 0)$ is a maximal order. From Proposition 3.10, 8 , it follows that various notions of a maximal order agree in our situation. So the main theorem of this note is proved in this case (for algebras $A(\alpha, \beta, 0)$ ). It should be noted that finiteness of order of $\theta$ does not force $\gamma=0$. For example, the algebra $A(-1,-1, \gamma)$ has a corresponding automorphism $\theta$ of order three for any $\gamma$. For prime rings, the hypothesis of stable freeness can be weakened by the hypothesis that the state space (see [9] for a definition) is trivial. Since orders in central algebras are prime (PI) rings (Corollary 13.6.6, 8]), this provides an alternate method to prove that the down-up algebras are maximal orders over their centers when $\theta$ is a finite order automorphism. However the purpose of this article to (re)introduce the method sketched above to questions related to maximality of orders. The method of the last paragraph is particularly useful in applications to crossed product algebras.

The results of this article complete the analysis in [7]. The main result of this article gives a family of explicit examples of maximal orders with three dimensional centers. Currently there is considerable activity in the area of maximal orders on algebraic surfaces, and so it is natural to consider similar questions for higher dimensional algebraic varieties. We hope these examples will be of interest in this context.

\section{Down-up ALgebras at RoOts OF UNITY}

In this section, we consider the case when the automorphism $\theta$ is of finite order $m$. Then the center $R$ of $A$ is generated by $\left\{u^{m}, d^{m}\right\}$ over $(\mathbb{C}[\xi, \eta])^{\theta}$ (Corollary 2.0.2, [7]). In this case, we have two polynomials $w_{i} \in \mathbb{C}[\xi, \eta], i=1,2$, such that

$$
\theta\left(w_{1}\right)=\lambda_{1} w_{1}, \theta\left(w_{2}\right)=\lambda_{2} w_{2}
$$

where $\lambda_{1}, \lambda_{2}$ are $l_{1}^{\text {th }}$ and $l_{2}^{\text {th }}$ roots of unity resp. and $m$ is the LCM of $l_{1}$ and $l_{2}$. It is easy to see that $\lambda_{i}$ 's are roots of the quadratic equation $q(t)=t^{2}+\frac{\alpha}{\beta} t-\frac{1}{\beta}$. We consider the commutative ring

$$
S=\frac{\mathbb{C}[\xi, \eta, \mu, \nu]}{\left(\xi \theta(\xi) \cdots \theta^{m-1}(\xi)-\mu \nu\right)}
$$


and extend the action of $\theta$ to $S$ by letting it act trivially on $\mu, \nu$. Define the homomorphism

$$
\begin{aligned}
S & \longrightarrow A, \\
\xi, \eta & \mapsto u d, d u \text { resp. } \\
\mu, \nu & \mapsto u^{m}, d^{m} \text { resp. }
\end{aligned}
$$

Note that this is an embedding of $S$ into $A$ (Lemma 6.1.1, 7]). We identify the image of this map and denote it by $S$ again. We start with an easy lemma.

Lemma 2.1. The algebra $S$ is a maximal commutative subalgebra of $A$.

Proof. Let $R^{\prime}$ denote the polynomial ring $\mathbb{C}[\xi, \eta]$ and let $R^{\prime}[u ; \theta]$ and $R^{\prime}\left[d ; \theta^{-1}\right]$ be skew polynomial rings. We first recall (Lemma 2.0.1, [7]) that the algebra $A$ is isomorphic to

$$
R^{\prime} \oplus u R^{\prime}[u ; \theta] \oplus d R^{\prime}\left[d ; \theta^{-1}\right]
$$

as an $R^{\prime}$-module. In particular, $A$ is a free $R^{\prime}$-bimodule with a basis given by the decomposition above. Let $p \in A$ be an element that commutes with $S$. We decompose this element by using the basis above. Note that since $R^{\prime} \subset S$, we may assume that

$$
p=\sum_{i \geq 1} a_{i} u^{i}+\sum_{j \geq 1} b_{j} d^{j} a_{i}, b_{j} \in R^{\prime} .
$$

Then

$$
\begin{aligned}
p \xi & =\sum_{i \geq 1} a_{i} u^{i} \xi+\sum_{j \geq 1} b_{j} d^{j} \xi \\
& =\sum_{i \geq 1} a_{i} \theta^{i}(\xi) u^{i}+\sum_{j \geq 1} b_{j} \theta^{-j}(\xi) d^{j} \\
\text { and } \xi p & =\sum_{i \geq 1} a_{i} \xi u^{i}+\sum_{j \geq 1} b_{j} \xi d^{j} .
\end{aligned}
$$

Since $R^{\prime}$ is a domain, by uniqueness of decomposition

$$
\theta^{i}(\xi)=\xi \text { whenever } a_{i} \neq 0 \text { or } b_{i} \neq 0 .
$$

Similarly

$$
\theta^{i}(\eta)=\eta \text { whenever } a_{i} \neq 0 \text { or } b_{i} \neq 0 .
$$

So $\theta^{i}=$ identity whenever either $a_{i}$ or $b_{i}$ is nonzero. So $i$ is divisible by $m$ whenever either $a_{i}$ or $b_{i}$ is nonzero. So

$$
p=\sum_{i \geq 1} a_{m i} u^{m i}+\sum_{j \geq 1} b_{m j} d^{m j}
$$

and hence it belongs to $S$. So $S$ is a maximal commutative subalgebra of $A$.

Next we prove another useful proposition.

Proposition 2.2. The algebra $S$ and hence $R$ are integrally closed in their respective fraction fields.

Proof. Let $Q(R), Q(S)$ denote the fraction fields of $R$ and $S$ respectively. Also let $S_{\mu}$ and $S_{\nu}$ denote the localizations of $S$ at the subsets $\left\{\mu^{i}\right\}_{i \geq 1}$ and $\left\{\nu^{i}\right\}_{i \geq 1}$. Then $S_{\mu}$ and $S_{\nu}$ are isomorphic to the localizations $\mathbb{C}[\xi, \eta, \mu]_{\mu}$ and $\mathbb{C}[\xi, \eta, \nu]_{\nu}$ resp. Hence they are integrally closed. If $\alpha \in Q(S)$ is a root of a monic polynomial 
$f$ with coefficients in $S$, then $\alpha \in S_{\mu} \cap S_{\nu}$. So it will be sufficient to prove that $S_{\mu} \cap S_{\nu}=S$.

To that end, let $\frac{\bar{f}}{\mu^{i}}=\frac{\bar{g}}{\nu^{j}}$ for some nonnegative integers $i, j$ and $f, g \in \mathbb{C}[\xi, \eta, \mu, \nu]$. The bar denotes the image in $S$. We use induction on $i+j$. Clearly the claim is true for $i+j=1$. Now let $i+j \geq 2$. We may assume that $i, j \neq 0$. Then

$$
\begin{aligned}
\nu^{j} \bar{f}-\mu^{i} \bar{g} & =0 \text { in } S \text { and hence } \\
\nu^{j} f-\mu^{i} g & =h\left(\xi \cdots \theta^{m-1}(\xi)-\mu \nu\right) \text { for some } h \in \mathbb{C}[\xi, \eta, \mu, \nu] .
\end{aligned}
$$

So it follows that $h=a \mu+b \nu$ for some $a, b \in \mathbb{C}[\xi, \eta, \mu, \nu]$. Expanding we get

$$
\left(\nu^{j} f+b \mu \nu^{2}\right)-\left(\mu^{i} g-a \mu^{2} \nu\right)=(a \mu+b \nu)\left(\xi \cdots \theta^{m-1}(\xi)\right) .
$$

Considering this equation $\bmod \mu$,

$$
\nu^{j} f=b \nu \xi \cdots \theta^{m-1}(\xi) \bmod \mu .
$$

So

$$
f=f_{1} \xi \cdots \theta^{m-1}(\xi)+f_{1}^{\prime} \mu \text { for some } f_{1}, f_{1}^{\prime} \in \mathbb{C}[\xi, \eta, \mu, \nu] .
$$

Now in $S$ this gives $\bar{f}=\bar{f}_{1} \mu \nu+\bar{f}_{1}^{\prime} \mu$. So $\bar{f}$ is divisible by $\mu$. Similarly $\bar{g}$ is divisible by $\nu$. By induction

$$
\frac{\bar{f}}{\mu^{i}}=\frac{\bar{g}}{\nu^{j}} \in S
$$

This finishes the proof that $S$ is integrally closed. Now consider the ring $R$. Since $R \subset S$ and $S$ is integrally closed, it follows that the integral closure of $R$ is equal to $S \cap Q(R)$. But since $S^{\theta}=R$, it follows that $R$ is integrally closed in its fraction field.

Let $p$ be a height one prime ideal in $R$. For later use, we compute the inertia groups for the extensions $(S)_{p}$ over $R_{p}$. Note that the ring $(S)_{p}$ is the semilocal ring obtained by localizing $S$ at the multiplicative subset $R-p$. Recall that we have polynomials $w_{1}, w_{2} \in \mathbb{C}[\xi, \eta]$ that are eigenvectors with eigenvalues $\lambda_{1}, \lambda_{2}$ resp. for the action of $\theta$ on (inhomogeneous) linear polynomials in $\mathbb{C}[\xi, \eta]$.

Lemma 2.3. Let $q$ be a maximal ideal of $(S)_{p}$ lying over the ideal $p$ of $R_{p}$. If the inertia group $G_{q}^{I}$ of $q$ is nontrivial, then either $q=\left(w_{1}\right)$ or $q=\left(w_{2}\right)$. In these cases, the inertia groups are generated by $\theta^{l_{2}}$ and $\theta^{l_{1}}$ resp. (Recall that $\lambda_{1}$ and $\lambda_{2}$ are $l_{1}^{\text {th }}$ and $l_{2}^{\text {th }}$ roots of unity.)

Proof. Let $q=\left(w_{1}\right)$. Then $q$ is totally ramified. Also $S_{q} / q S_{q}$ is isomorphic to the field of rational functions $\mathbb{C}\left(\mu, w_{2}\right)$. It follows that the inertia group is as above, as the stabilizer subgroup of $w_{2}$ is generated by $\theta^{l_{2}}$ and $\theta$ acts trivially on $\mu$. The other case follows as well. This finishes the second part.

Now we consider the first statement. We first compute the differents $\mathcal{D}\left(S_{q} / R_{p}\right)$ when $w_{i} \notin q$ for $i=1,2$. We use the following fact about differents. Let $L=K(\alpha)$ be a separable field extension of degree $n$, and let $A$ be a Dedekind domain with fraction field $K$. Suppose that $\alpha$ is integral over $A$ with minimal polynomial $f(x) \in$ $A[x]$. Let $B$ be the integral closure of $A$ in $L$. Then the different of $B / A$ divides the ideal $\left(f^{\prime}(\alpha)\right)$ where $f^{\prime}(x)$ is the formal derivative of $f(x)$.

Choose a pair of integers $(i, j)$ so that $\lambda_{1}^{i} \lambda_{2}^{j}$ is a primitive $m^{\text {th }}$ root of unity. We use the fact above with $\alpha=w_{1}^{i} w_{2}^{j}$. A standard calculation shows that $\alpha$ generates $Q\left((S)_{p}\right)$ over $Q\left(R_{p}\right)$ and that the minimal polynomial of $\alpha$ is $f(x)=x^{m}-\alpha^{m}$. So $\mathcal{D}\left(S_{q} / R_{p}\right)$ divides the ideal $\left(\left(w_{1}^{i} w_{2}^{j}\right)^{m-1}\right)$. Since $w_{i} \notin q$ for $i=1,2$, it follows that 
$\mathcal{D}\left(S_{q} / R_{p}\right)=S_{q}$. This means that the inertia group $G_{q}^{I}$ is trivial in these cases. This finishes the lemma.

Next we recall a criterion due to $\mathrm{S}$. Williamson for maximality of orders $([10])$. Let $B$ be a tamely ramified extension of a rank one discrete valuation $\operatorname{ring} A$ such that the quotient field extension is finite Galois with Galois group $G$. We assume that $B$ is also a discrete valuation ring, as this is the only case we will need below. Let $G^{I}$ be the inertia group of $B$ over $A$. Let $K$ be the residue field of $B$ and let $k$ be the residue field of $A$. Let $\Delta(f, B, G)$ be the crossed product associated to a cocycle $f$ with cohomology class in $H^{2}\left(G, B^{\times}\right)$. Define the conductor group $H_{f}$ to be the maximal subgroup of $G^{I}$ such that $[\bar{f}]$ is in the image of the inflation map $H^{2}\left(G / H_{f}, K^{\times}\right) \rightarrow H^{2}\left(G, K^{\times}\right)$. Then S. Williamson's criterion (Thm. 2.5, [10], together with Thm. 3.3, 4]), says that the order $\Delta(f, B, G)$ is maximal if and only if the conductor group is trivial.

We also define the group $\Gamma_{f}$ to be the maximal subgroup of $G^{I}$ such that the image of class of $[\bar{f}]$ under the restriction map $H^{2}\left(G, K^{\times}\right) \rightarrow H^{2}\left(\Gamma_{f}, K^{\times}\right)$is trivial. Since the composition map

$$
H^{2}\left(G / H_{f}, K^{\times}\right) \longrightarrow H^{2}\left(G, K^{\times}\right) \longrightarrow H^{2}\left(\Gamma_{f}, K^{\times}\right)
$$

is trivial, $H_{f} \subset \Gamma_{f}$.

Now we apply these considerations to our case. First we recall that certain localizations of the down-up algebra are crossed products. Namely, consider localizations $A_{\mu}$ and $A_{\nu}$ of $A$ at $\left\{\mu^{i}\right\}$ and $\left\{\nu^{j}\right\}$ respectively. Also we consider two crossed products

$$
\Delta_{\mu}=\Delta\left(S_{\mu}, f_{\mu}, G\right), \Delta_{\nu}=\Delta\left(S_{\nu}, f_{\nu}, G\right),
$$

where $G$ is the cyclic group of automorphisms of $S_{\mu}$ and $S_{\nu}$ generated by $\theta$ and $f_{\mu}, f_{\nu}$ are the cocycles

$$
f_{\mu}\left(\theta^{i}, \theta^{j}\right)=\left\{\begin{array}{ll}
\mu & i+j \geq m \\
1 & i+j<m
\end{array} \quad f_{\nu}\left(\theta^{-i}, \theta^{-j}\right)=\left\{\begin{array}{cc}
\nu & i+j \geq m \\
1 & i+j<m
\end{array} .\right.\right.
$$

Then we have proved (Theorem 6.1.1, [7]) that the algebra $A_{\mu}$ (resp. $A_{\nu}$ ) is isomorphic to $\Delta_{\mu}$ (resp. $\Delta_{\nu}$ ). We use these crossed product structures in the following paragraphs.

Let $p$ be a codimension one prime in $R$. Suppose $q$ is the only prime in $S_{\mu}$ lying over $p$ (so that $q=\left(w_{1}\right)$ or $\left(w_{2}\right)$ ). Let $G_{q}$ be the decomposition subgroup of $q$ in $G$ and let

$$
f_{p}: G \times G \longrightarrow\left(S_{q}\right)^{\times}
$$

be the cocycle describing the crossed product structure of $A_{p}$ (that is, $A_{p}=$ $\left.\Delta\left(S_{q}, f_{p}, G\right)\right)$. Let $K$ denote the field $Q\left(S_{q} / q S_{q}\right)$. Note that in our case the decomposition group of $q$ is $G$. We construct a new cocycle $\bar{f}_{p}$,

$$
\bar{f}_{p}: G \times G \longrightarrow K^{\times},
$$

in an obvious way. We have the following lemma.

Lemma 2.4. Let $p$ be a codimension one prime in $R$. Then for the cocyle $f_{p}$ the subgroups $\Gamma_{f_{p}}$ and hence $H_{f_{p}}$ are trivial.

Proof. We first prove the lemma in the case when the codimension one prime $q=$ $\left(w_{1}\right)$ or $\left(w_{2}\right)$ lies over $p$. We may assume $q=\left(w_{1}\right)$, as the other case follows by a similar calculation. We show that the restriction of $\bar{f}_{p}$ in $H^{2}\left(G^{\prime}, K^{\times}\right)$for any 
subgroup $G^{\prime}$ of $G_{q}^{I}$ is trivial only if $G^{\prime}$ is the trivial subgroup. In this case, the inertia group $G_{q}^{I}=\left(\theta^{l_{2}}\right)$ and so a subgroup $G^{\prime}$ is generated by $\theta^{l_{2} n}$ for some integer $n$. Recall that the linear polynomials $w_{1}, w_{2}$ are the eigenvectors for the action of $\theta$ on the (affine) linear part of the polynomial ring $\mathbb{C}[\xi, \eta]$. In this notation, it is easy to see that the residue field $K$ is the fraction field

$$
\left(\frac{\mathbb{C}\left[w_{2}, \mu, \nu\right]}{\left(\prod_{i}\left(\frac{-\lambda_{2}^{i} w_{2}}{\lambda_{1}-\lambda_{2}}-\frac{\gamma}{\beta\left(\lambda_{1}-1\right)\left(\lambda_{2}-1\right)}\right)\right)-\mu \nu}\right) .
$$

Here we have assumed that the eigenvalues are distinct from 1 . The only remaining possibility is when one of the eigenvalues is 1 . This happens only if theta is of order two; that is, the other eigenvalue is -1 and $\gamma=0$. In this case, the algebra is $A(0,1,0)$ and the eigenvectors $w_{1}, w_{2}$ are

$$
w_{1}=\xi+\eta, \quad w_{2}=\xi-\eta .
$$

This gives that the field $K$ in this case is $K=\left(\frac{\mathbb{C}\left[w_{1}, \mu, \nu\right]}{\left(w_{1}^{2}-4 \mu \nu\right)}\right)$. Consider the restriction of the cocycle $\bar{f}_{p}$ to $G^{\prime} \times G^{\prime}$. If the corresponding cohomology class is trivial, then there exists a cochain $h_{p}: G^{\prime} \rightarrow K^{\times}$such that $d h_{p}=\bar{f}_{p}$. That is,

$$
\frac{\left(\theta^{n l_{2}}\right)^{i_{1}}\left(h_{p}\left(\left(\theta^{n l_{2}}\right)^{i_{2}}\right)\right) h_{p}\left(\left(\theta^{n l_{2}}\right)^{i_{1}}\right)}{h_{p}\left(\left(\theta^{n l_{2}}\right)^{i_{1}+i_{2}}\right)}=\left\{\begin{array}{ll}
1 & i_{1}+i_{2}<\frac{m}{n l_{2}} \\
\mu & i_{1}+i_{2} \geq \frac{m}{n l_{2}}
\end{array} .\right.
$$

Note that $\theta^{l_{2}}$ acts trivially on $K$, and so this condition is equivalent to the condition that $\mu \in\left(K^{\times}\right)^{\frac{m}{n l_{2}}}$. The defining polynomial for the field $K$ is

$$
(-1)^{m}\left(\frac{w_{2}^{l_{2}}}{\left(\lambda_{1}-\lambda_{2}\right)^{l_{2}}}+\frac{\gamma^{l_{2}}}{\beta^{l_{2}}\left(\lambda_{1}-1\right)^{l_{2}}\left(\lambda_{2}-1\right)^{l_{2}}}\right)^{\frac{m}{l_{2}}}-\mu \nu .
$$

It follows that $\mu$ is not an $\left(\frac{m}{n l_{2}}\right)$-th root unless $n l_{2}=m$, which implies that $G^{\prime}$ is trivial. This shows that the group $\Gamma_{f_{p}}$ is trivial. Since the conductor group $H_{f_{p}} \subset \Gamma_{f_{p}}$, it follows that it is also trivial. This finishes the lemma.

Remark 2.5. By Lemma 2.3, when $q \neq\left(w_{1}\right)$ or $\left(w_{2}\right), G_{q}^{I}$ is trivial. So in this case the conductor group for the corresponding cocycle is trivial as well. For the definition of conductor a group when the extension of the discrete valuation ring is not itself a discrete valuation ring, see the discussion preceding Thm. 2.5, [10].

Theorem 2.6. Let the automorphism $\theta$ be of finite order $m$. Then $A$ is a maximal $R$-order in $A \otimes_{R} Q(R)$.

Proof. First note that

$$
A \otimes_{R} Q(R) \cong A_{\mu} \otimes_{R_{\mu}} Q\left(R_{\mu}\right) \cong \Delta_{\mu} \otimes_{R_{\mu}} Q\left(R_{\mu}\right) \cong \Delta\left(S_{\mu} \otimes_{R \mu} Q\left(R_{\mu}\right), f_{\mu}, G\right) .
$$

Now $S_{\mu}$ is integral over $R_{\mu}$, and so $S_{\mu} \otimes_{R_{\mu}} Q\left(R_{\mu}\right)$ is a field, isomorphic to $Q\left(S_{\mu}\right)$. So $A \otimes_{R} Q(R)$ is a central simple algebra. Since $A$ is a finitely generated $R$-module, $A$ is an $R$-order in $A \otimes_{R} Q(R)$.

We recall a theorem of Auslander-Goldman (Thm. 1.5, [1]) which gives a characterization of maximal orders. It says that an $R$-order $\Lambda$ is maximal if and only if it is a reflexive $R$-order such that for any codimension 1 prime $p$ of $R, \Lambda_{p}$ is a maximal $R_{p}$-order. Recall that an $R$-order $\Lambda$ is reflexive if $\Lambda^{* *}=\Lambda$ where $\Lambda^{*}=\operatorname{Hom}_{R}(\Lambda, R)$. If $R$ is integrally closed, then $\Lambda^{* *}=\bigcap \Lambda_{p}$, where the intersection is taken over all codimension 1 primes of $R$. 
In order to prove that $A$ is a reflexive $R$-order, we first show that $A_{\mu} \cap A_{\nu}=A$ in $A \otimes_{R} Q(R)$. Since $A$ is a domain, $A \subset A_{\mu} \cap A_{\nu}$. Let

$$
\frac{\sum a_{i j k} u^{i}(d u)^{j} d^{k}}{u^{m \alpha}}=\frac{\sum b_{i^{\prime} j^{\prime} k^{\prime}} u^{i^{\prime}}(d u)^{j^{\prime}} d^{k^{\prime}}}{d^{m \beta}}
$$

for some positive integers $\alpha, \beta$ and $a_{i j k}, b_{i^{\prime} j^{\prime} k^{\prime}} \in \mathbb{C}$. Note that the numerators of these expressions are uniquely determined by the PBW basis theorem. Comparing coefficients, the same theorem gives

$$
\begin{gathered}
a_{i j k}=b_{i^{\prime} j^{\prime} k^{\prime}} \text { if and only if } i=i^{\prime}+m \alpha, j=j^{\prime}, k=k^{\prime}-m \beta, \text { and } \\
i \geq m \alpha, k^{\prime} \geq m \beta \text { for all nonzero coefficients } a_{i j k}, b_{i^{\prime} j^{\prime} k^{\prime}} .
\end{gathered}
$$

So the element in Eq. (2.1) is in $A$. Now we show that $\bigcap A_{p}=A_{\mu} \cap A_{\nu}$, where the intersection is taken over all codimension one primes in $R$. Observe that a codimension one prime $p$ of $R$ cannot contain both $\mu$ and $\nu$. So it will be sufficient to show that

$$
A_{\mu}=\bigcap A_{p} \text { and } A_{\nu}=\bigcap A_{q}
$$

where the first (resp. second) intersection is taken over all codimension one primes not containing $\mu$ (resp. $\nu$ ). Note that proving these equalities is the same as proving that $A_{\mu}$ and $A_{\nu}$ are reflexive orders. We show one of these equalities, as the other is similar.

Recall that $A_{\mu}$ is isomorphic to the crossed product algebra $\Delta_{\mu}$ described above. So it will be sufficient to show that $\Delta_{\mu}$ is a reflexive order. First we note that as an $S_{\mu}$ module, $\Delta_{\mu}$ is free of rank equal to the order of $\theta$. So the reflexivity of $\Delta_{\mu}$ follows from that of $S_{\mu}$. Since $R_{\mu}$ is integrally closed, it is sufficient to show that

$$
S_{\mu}=\bigcap\left(S_{\mu}\right)_{p}=(S)_{p},
$$

where the intersection is taken over codimension one primes of $R$ not containing $\mu$. Note that the multiplicative subset in each of these localizations is $R-p$. So

$$
(S)_{p} \subset \bigcap_{i} S_{p_{i}},
$$

where the $p_{i}$ 's are primes in $S$ lying over the prime $p$ of $R$. Note that the multiplicative subset in localization $S_{p_{i}}$ is $S-p_{i}$. Thus it follows that

$$
\bigcap\left(S_{\mu}\right)_{p} \subset \bigcap_{p} S_{i} S_{p_{i}}
$$

where on the right side the first intersection is over codimension one primes $p$ of $R$ not containing $\mu$ and the second intersection is over all primes $p_{i}$ of $S$ lying over $p$. Now since $S_{\mu}$ is normal and $S_{\mu}$ is a finite module over $R_{\mu}$, it follows that

$$
\bigcap_{p} \bigcap_{i} S_{p_{i}}=S_{\mu}
$$

So we conclude that $S_{\mu}$ is reflexive over $R_{\mu}$, and hence so is $\Delta_{\mu}$. This finishes the proof that $A$ is a reflexive $R$-order.

It remains to consider maximality of $A_{p}$ as an $R_{p}$-order for any codimension one prime $p$ of $R$. Since a codimension one prime cannot contain both $\mu$ and $\nu, p$ is a codimension one prime in $R_{\mu}$ or $R_{\nu}$. Thus $A_{p}$ is also a crossed product order. We assume that $\mu$ is not in $p$, as the other case is similar. By S. Williamson's criterion above, $A_{p}$ is a maximal order if and only if the corresponding conductor group $H_{f_{p}}$ 
is trivial. If $q=\left(w_{1}\right)$ or $\left(w_{2}\right)$ does not lie over $p$, then by Remark 2.5, the conductor group is trivial. So $A_{p}$ is a maximal order for such a prime $p$.

If $q=\left(w_{1}\right)$ or $\left(w_{2}\right)$ lies over $p$, then by Lemma 2.4 the conductor group $H_{f_{p}}$ is trivial. So the order $A_{p}$ is maximal by the criterion of S. Williamson. Since localizations of $A$ at codimension one primes are maximal and $A$ is a reflexive order, it follows that the algebra $A$ is a maximal order in $A \otimes_{R} Q(R)$. This finishes the proof of the theorem.

\section{ACKNOWLEDGMENTS}

We thank Georgia Benkart for her interest and useful comments. We also thank the referee for pointing out a crucial mistake in an earlier version of this article.

\section{REFERENCES}

[1] Auslander, M., and Goldman, O., Maximal orders, Trans. Amer. Math. Soc. 97 (1960) 1-24. MR0117252(22:8034)

[2] Benkart, G., and Roby, T., Down-up algebras, J. Algebra 209 (1998) 305-344. MR.1652138 (2000e:06001a)

[3] Benkart, G., and Witherspoon, S., A Hopf structure for down-up algebras, Math. Z. 238 (2001) 523-553. MR1869697 (2002k:16059)

[4] Harada, M., Hereditary orders, Trans. Amer. Math. Soc. 107 (1963) 273-290. MR0151489 $(27: 1474)$

[5] Kirkman, E., and Musson, I., Hopf down-up algebras, J. Algebra 262 (2003) 42-53. MR1970801 (2004c:16065)

[6] Kirkman, E., Musson, I., and Passman, D., Noetherian down-up algebras, Proc. Amer. Math. Soc. 127 (1999) 3161-3167. MR1610796 (2000b:16042)

[7] Kulkarni, R. S., Down-up algebras and their representations, J. Algebra 245 (2001) 431-462. MR:1863888 (2002k:16061)

[8] McConnell, J. C., and Robson, J. C., Noncommutative Noetherian Rings, John Wiley and Sons, 1987. MR934572 (89j:16023)

[9] Stafford, J. T., Auslander-regular algebras and maximal orders, J. London Math. Soc. (2) 50 (1994) 276-292. MR:1291737 (95j:16028)

[10] Williamson, S., Crossed products and hereditary orders, Nagoya Math. J. 23 (1963) 103-120. MR0163943 (29:1242)

Department of Mathematics, Wells Hall, Michigan State University, East Lansing, MiCHIGAN 48824

E-mail address: kulkarni@math.msu.edu 